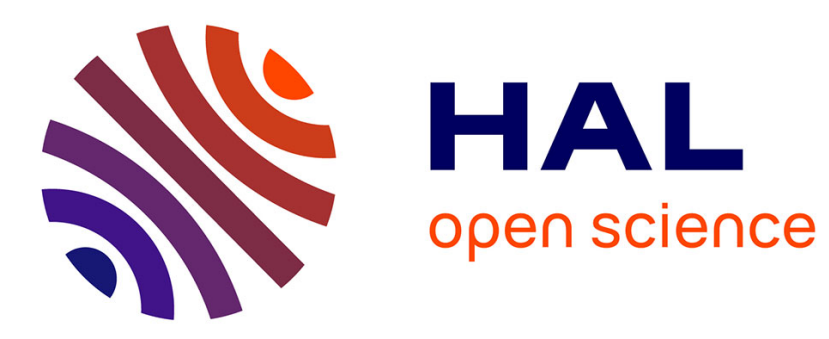

\title{
Assessing the Versatility of Molecular Modelling as a Strategy for Predicting Gas Adsorption Properties of Chalcogels
}

Irene Amiehe Amiehe Essomba, Carlo Massobrio, Mauro Boero, Guido Ori

\section{- To cite this version:}

Irene Amiehe Amiehe Essomba, Carlo Massobrio, Mauro Boero, Guido Ori. Assessing the Versatility of Molecular Modelling as a Strategy for Predicting Gas Adsorption Properties of Chalcogels. Theory and Simulation in Physics for Materials Applications, pp.23-37, 2020, 10.1007/978-3-030-37790-8_2 . hal-03036655

\section{HAL Id: hal-03036655 \\ https://hal.science/hal-03036655}

Submitted on 2 Dec 2020

HAL is a multi-disciplinary open access archive for the deposit and dissemination of scientific research documents, whether they are published or not. The documents may come from teaching and research institutions in France or abroad, or from public or private research centers.
L'archive ouverte pluridisciplinaire HAL, est destinée au dépôt et à la diffusion de documents scientifiques de niveau recherche, publiés ou non, émanant des établissements d'enseignement et de recherche français ou étrangers, des laboratoires publics ou privés. 


\title{
Assessing the versatility of molecular modelling as a strategy for predicting gas adsorption properties of chalcogels
}

\author{
Irene Amiehe Essomba Berenger ${ }^{\mathrm{a}}$, Carlo Massobrio $^{\mathrm{a}}$, Mauro Boero $^{\mathrm{a}}$, Guido Ori*a \\ ${ }^{a}$ Université de Strasbourg, CNRS, Institut de Physique et Chimie des Matériaux de Strasbourg, UMR 7504, F-67034 Strasbourg, France
}

\begin{abstract}
Modelling gas adsorption of porous materials is nowadays an undeniable necessary in order to complement experiment findings with the purpose to enrich our fundamental understanding of adsorption mechanisms as well as develop better performing materials for gas mixture separation. In this contribution, we explore the possibility to use first-principles molecular dynamics (FPMD) and grand canonical Monte Carlo (GCMC) simulations to target the gas adsorption of disordered nanoporous chalcogenides (i.e. chalcogels). This computational scheme allows us to take advantage of the ability of FPMD to accurately describe the structure and bonding of the disordered nature of chalcogels as well as the potential of GCMC to model the adsorption mechanisms of porous networks. We assess the versatility of such scheme by evaluating the role of pore size, chemical stoichiometry and composition for multiple chalcogenide-based systems on nitrogen adsorption isotherms.
\end{abstract}

Keywords: first-principles molecular dynamics, porous materials, chalcogel, gas adsorption, grand canonical Monte Carlo, chalcogenide glasses

\section{Introduction}

The development of porous materials for gas adsorption, separation and purification is central to many energy and 
for this class of porous materials, the study of their adsorption properties is still limited.[26, 27, 28, 29] While adsorption and transport in porous materials such as zeolites, porous carbon and MOF are relatively well understood, the specific case of chalcogels remains unclear with many questions left unexplained such as the role of the surface chalcogenide chemistry and the specific interactions playing at the gas/solid interface in the confined environment. This arises from a general limitation of the experimental studies of gas adsorption of chalcogels as most methods are unable to detect the features of this buried gas/solid interface. In this respect, molecular modelling has proven to be an efficient technique to investigate the gas adsorption of such porous materials. In few recent works, we resorted on a computational scheme based on first-principles molecular dynamics (FPMD) and grand canonical Monte Carlo simulations (GCMC) to identify the microscopic mechanisms of $\mathrm{N}_{2}, \mathrm{CO}_{2}, \mathrm{H}_{2}$ and $\mathrm{CH}_{4}$ adsorption in a prototypical chalcogel made of glassy $\mathrm{GeS}_{2}\left(g-\mathrm{GeS}_{2}\right.$ hereafter).[30, 31] With the present contribution we aim to widen this first work and to assess the versatility of such computational scheme to gain insights on the role of pore size, chemical stoichiometry and composition for multiple chalcogenide-based systems on nitrogen adsorption isotherms. Gas adsorption of simple fluids such as $\mathrm{N}_{2}$ at $77 \mathrm{~K}$ is a routine technique which allows characterizing the specific surface, porous volume, and pore size distribution of microporous and mesoporous media. This contribution is organized as follows. In a first section, the main advantages of employing FPMD and GCMC simulations within the field of modelling chalcogels are presented. In a second section, as case studies, the role of pore size, chemical stoichiometry and compositions are analyzed and discussed. We critically assess our results by invoking available experimental data before drawing a final set of conclusions and perspectives.

\section{Computational methodology}

The computational scheme proposed here is based on the employment of FPMD simulations where the electronic structure is described within the framework of the density functional theory (DFT) and it allows to accurately describe the chemistry and bonding of amorphous chalcogenides and GCMC simulations to model the adsorption mechanisms of porous chalcogels. In Figure 1 a schematic cartoon of the four main building blocks of this methodology is presented:

1. Producing of a chalcogenide glass structural model in quantitative agreement with experimental findings;

2. Building and refinement of chalcogenide surface models at finite conditions;

3. Modelling gas adsorption mechanisms of chalcogel with defined porosity;

4. Detailed analysis of the gas/solid interface chemistry and computation of other properties.

Three out of the four building blocks resort to FPMD simulations in order to produce realistic and reliable structural models of glassy chalcogenides as well as being able to accurately describe the chemical interactions involved at 
- Producing a reliable chalcogenide glass model

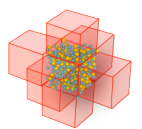

2- Building a realistic glass surface

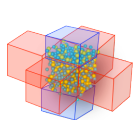

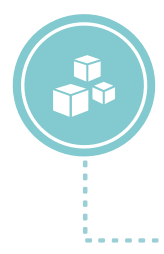

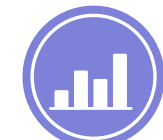

3- Modelling gas adsorption
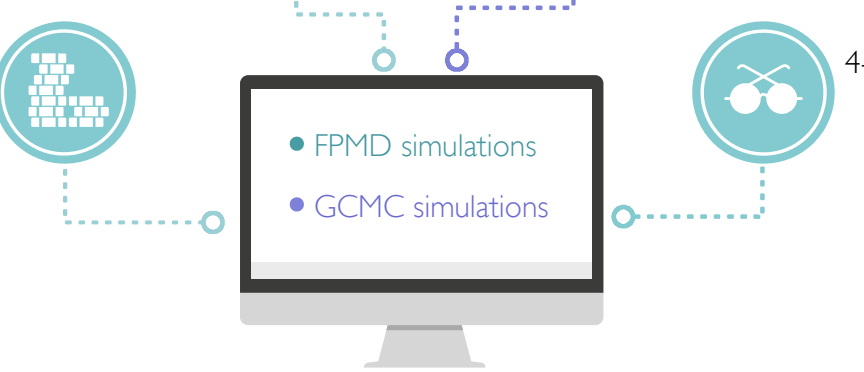

4- Interface chemistry and other properties

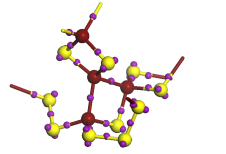

Figure 1: Schematic representation of the computational methodology for modelling gas adsorption of chalcogel materials.

4

\subsection{First-principles molecular dynamics: quantitative prediction of structure and bonding of bulk disordered chalco-} genides and their surfaces

In this section we tentatively summarize the reasons of first-principles molecular dynamics (FPMD)[34] combined with density functional theory (DFT) as the computational method of choice for the proper description of the chemistry and structure of disordered chalcogenides. Empirical interatomic potentials, routinely employed in classical MD simulations, are found to generally fail in reproducing the correct chemical behavior for systems other than those characterized by chemically ordered topologies (typical of oxide-based materials) as well as the presence of structural defects such as miss-coordinations and homopolar bonding. Thanks to a proper description of the local electronic structure, FPMD allows instead to face the hurdle of describing the complex interplay between different bonding interactions particular of disordered chalcogenide-based materials, whether liquids or glasses (such as ionocovalency[35] and metavalent bonding[36] among others). Typically, glassy phases are created by means of FPMD mimicking the melt-to-quench process used in the laboratory. Although employing a different time scale with respect to the experiments, FPMD simulations allow nowadays to achieve a quantitative structural description of disordered chalcogenides. This performance, accurately reproducing experimental data, arises from a proper choice of the FPMD computational scheme details such as the type of exchange-correlation (XC) functional, the role of dispersion forces (such as van der Waals (vdW)) and the size and length of the system and simulation trajectory, respectively. For the class of $\mathrm{Ge}-\mathrm{X}$ based chalcogenide glasses with $\mathrm{X}$ being the chalcogen counter-ion ( $\mathrm{S}, \mathrm{Se}$ or $\mathrm{Te}$ ), the generalized gradient approximation (GGA) scheme together with the XC functional developed by Becke,[37] Yang, and Parr[38] 
Table 1: Details of the bulk chalcogenide glasses produced by FPMD and considered in this work.

\begin{tabular}{cccccc}
\hline $\begin{array}{c}\text { chemical } \\
\text { composition }\end{array}$ & stoichiometry & n. atoms & $\begin{array}{c}\text { box size } L \\
(\AA)\end{array}$ & $\begin{array}{c}\text { density } \rho \\
\left(\text { atoms } \AA^{-3}\right)\end{array}$ & reference \\
\hline $\mathrm{g}-G e_{x} \mathrm{~S}_{y}$ & $\mathrm{GeS}_{4}$ & 480 & 24.845 & 0.0313 & {$[42]$} \\
$\mathrm{g}^{-G e} \mathrm{Se}_{y}$ & $\mathrm{GeSe}_{2}$ & 480 & 24.495 & 0.0327 & {$[43]$} \\
& $\mathrm{GeSe}_{4}$ & 480 & 24.871 & 0.0312 & {$[42]$} \\
& $\mathrm{GeSe}_{9}$ & 260 & 19.900 & 0.0330 & {$[44]$} \\
${\mathrm{g}-\mathrm{Ge}_{x} \mathrm{Te}_{y}}^{\mathrm{GeTe}_{4}}$ & 215 & 19.234 & 0.0302 & {$[41]$} \\
\hline
\end{tabular}

(BLYP) has been found to offer quantitative description (>96\%) for multiple structural properties (such as neutron and X-ray structure factors, total and partial pair correlation functions). This capability arises from the ability of BLYP to enhance the valance electron cloud localization of the local atomic arrangements with respect to other XC (Perdew, Becke and Ernzehof (PBE)[39, 40] for instance).[41] Table I shows the details of the systems considered in the present work and for which a quantitative agreement with experimental data was found over last few years. The specifics of the computational methodology employed for each system can be found in the references reported in Table I. The role of vdW interactions has also be taken into attentive account, especially if the interest is towards the study of the interactions of chalcogenide surfaces with other chemicals (such as gas or organic molecules). Multiple mathematical formalisms are available to describe the vdW interactions based on empirical corrections or on first-principles calculations making use of the electronic structure provided by DFT. However, regarding the specific case of disordered chalcogenides, a thoughtful choice has to be undertaken with respect to vdW formalism used depending on the type of system and chalcogen under study.[35, 45, 46] Typically a glass model is obtained by means of FPMD simulations within about $\sim 100-300$ ps of thermal treatment plus few tens of ps of equilibration at the final finite temperature of interest. This time frame is often statistically adequate to obtain a realistic structural model of the chalcogenide glass of interest. Longer equilibration time can be eventually needed for the computation of other properties (electronic, vibrational and thermal). Once the bulk chalcogenide glass model is obtained, the corresponding surface model can be built by opening to a vacuum space the unit cell box along one direction and properly relax its structure at finite temperature. This thermal treatment is needed in order allow surface local atomic rearrangements and structurally relax the atoms at the surface. In so doing, the presence of dangling bonds is reduced to a minimum. Table II shows the local and average coordinations around the atomic species for the surface models of the chalcogenides targeted in this work. The reported values are evaluated by considering the whole systems and have been calculated including neighbors separated by a cutoff corresponding to the first minimum in the partial pair correlation functions. All the systems considered are mainly made of fourfold coordinated Ge and twofold coordinated X atoms. However, these surface models show an increase in Ge and $\mathrm{X}$ atoms under- and over-coordinations contents at the expenses of the 
Table 2: Local atomic coordinations and charges of the glassy chalcogenide surfaces considered in this work. For completeness, polarizability and electronegativity values are also reported.

\begin{tabular}{|c|c|c|c|c|c|c|}
\hline \multirow[b]{2}{*}{ species } & \multirow[b]{2}{*}{ coordination } & \multicolumn{5}{|c|}{ local coordination distribution $(\%)^{a} /$ charge $q(e)^{b}$} \\
\hline & & $g-\mathrm{GeS}_{4}$ & $g-\mathrm{GeSe}_{2}$ & $g-\mathrm{GeSe}_{4}$ & $g-\mathrm{GeSe}_{9}$ & $g-\mathrm{GeTe}_{4}$ \\
\hline \multirow[t]{4}{*}{$\mathrm{Ge}$} & II & $6.3 / 0.99$ & $6.9 / 0.57$ & $10.4 / 0.76$ & $11.5 / 0.68$ & - \\
\hline & III & $11.5 / 1.02$ & $19.4 / 0.62$ & $21.9 / 0.78$ & 15.4 / 0.79 & $18.6 / 0.58$ \\
\hline & IV & $66.7 / 1.09$ & $58.8 / 0.72$ & $56.3 / 0.78$ & $73.1 / 0.94$ & $62.8 / 0.59$ \\
\hline & $\mathrm{V}$ & $15.6 / 1.01$ & $10.0 / 0.73$ & $9.4 / 0.84$ & - & $14.0 / 0.59$ \\
\hline \multirow[t]{3}{*}{$X$} & $\mathrm{I}$ & $8.3 /-0.29$ & $12.5 /-0.30$ & $12.5 /-0.19$ & $7.3 /-0.10$ & $14.5 /-0.13$ \\
\hline & II & $91.1 /-0.26$ & $67.8 /-0.34$ & $62.0 /-0.20$ & $88.9 /-0.10$ & $52.9 /-0.14$ \\
\hline & III & $0.5 /-0.51$ & 18.4 / -0.38 & $22.9 /-0.19$ & $3.0 /-0.13$ & $29.1 /-0.17$ \\
\hline \multirow[t]{3}{*}{$\bar{n} / \bar{q}^{c}$} & $\mathrm{Ge}$ & 3.92 / 1.06 & 3.57 / 0.66 & 3.58 / 0.77 & 3.62 / 0.89 & 3.77 / 0.56 \\
\hline & $\mathrm{X}$ & $1.92 /-0.27$ & $2.03 /-0.34$ & $2.05 /-0.19$ & $1.94 /-0.10$ & $2.08 /-0.14$ \\
\hline & $\mathrm{Ge}$ & S & & $\mathrm{Se}$ & & $\mathrm{Te}$ \\
\hline$\alpha{\text { (a.u. })^{d}}^{d}$ & 42 & 19 & & 27 & & 40 \\
\hline E.N. ${ }^{e}$ & 2.01 & 2.5 & & 2.4 & & 2.01 \\
\hline
\end{tabular}

a Atomic coordinations are calculated including neighbors separated by a cutoff corresponding to the first minimum of the corresponding partial pair correlation functions. ${ }^{b}$ The atomic charges $q$ are calculated by using the Qeq method developed by Rappé and Goddard et al. [47]. ${ }^{c}$ Polarizability values are obtained from [48]. ${ }^{d}$ Electronegativity values are obtained from [49].

fourfold and twofold coordinations with respect to the bulk counterparts. These results show that the surface models show a slightly lower chemical order than the bulk counterparts. It is important to highlight that the decrease of the chemical order degree characterizes the external layers of the surface models (about $\sim 7-8 \AA$ from the surface), whereas in the inner-region the atom species keeps and/or recover coordination distributions typical of bulk models. As an example, the inner-region region of $g-\mathrm{GeSe}_{2}$ surface model shows a $\sim 73 \%$ of Ge atoms fourfold coordinated and $\sim 86 \%$ of Se atoms twofold coordinated, which is inline with the contents found in the parent bulk model ( $\sim 76 \%$ and $\sim 93 \%$, respectively). Regarding the analysis of chemical bonding, both the electron localization function (ELF) and the maximally localized Wannier functions (MLWF) schemes are nowadays two widely used methodologies to study the valence electron (de)localization and structure and infer on the type of chemical interactions involved in amorphous chalcogenides. As an archetypal example, herein we report the typical information that can be extracted resorting to the MLWF formalism, based on the notion of Wannier functions, the MLWF centers (WFCs) and their spread. This scheme allows to seek further insight into the interplay between atomic structure and electronic properties with respect to the standard information based on the electronic density of states. Recently, we have employed these quantities to rationalize the extent of covalent $v$ s ionic nature of bonding for $g-\mathrm{GeS}_{4}, g-\mathrm{GeSe}_{4}$ and $g$-GeTe $\mathrm{Ge}_{4}$ This has ben achieved by relying on the correspondence between the distances identified in the atom-WFCs pair correlation functions $\left(g_{\alpha \beta}(r)\right.$, with $\alpha=\mathrm{X}$ and $\beta=$ WFCs). The data $\left(g_{X W}(r)\right.$ and WFCs spread) considered in the following discussion can found be found in ref. [41] and [42]. In particular, three types of WFCs can be distinguished in this series of binary glassy chalcogenides. The first type, labeled $\mathrm{W}_{b}$, represents a clear fingerprint of Ge-X heteropolar 
bonding and it is identified as the $g_{X W}(r)$ second peak found at about $\sim 0.9 \AA, \sim 1 \AA$ and $\sim 1.25 \AA$ for $g$-GeS $\mathrm{G}_{4}, g$-GeSe 4 and $g-\mathrm{GeTe}_{4}$, respectively. The second type, labeled $\mathrm{W}_{h}$, refers to homopolar X-X bonding and it corresponds to the typical $g_{X W}\left(r\right.$ third peak: $\mathrm{W}_{h}$ is found at about $\sim 1.02 \AA, \sim 1.18 \AA$ and $\sim 1.4 \AA$ for $g-\mathrm{GeS}_{4}, g-\mathrm{GeSe}_{4}$ and $g-\mathrm{GeTe}_{4}$, respectively. The third type, labeled $\mathrm{W}_{l p}$, refers to lone pair valence electrons not participating directly to chemical bonds but remaining localized in the proximity of the $\mathrm{X}$ atoms. Given the above definitions, the locations of the $\mathrm{W}_{b}$ centers with respect to the $\mathrm{X}$ atoms can be used to compare the covalency $v s$ ionicity degree of bonding that characterizes these glasses. The relative position of the $g_{X W}(r)$ second peak and the closer location to its origin allow to quantify the greater ionic character of the Ge-X covalent bonds along the serie: $g$-GeS $4>g-\mathrm{GeSe}_{4}>g-\mathrm{GeTe}_{4}$. This stems from the fact that the centers of valence electronic localization (related to heterogeneous bonding) are systematically closer to the S sites with respect to Ge sites than to the Se and Te sites, respectively. Furthermore, the inspection of the electronic (de)localization degree (spread, $\omega$ ) of the WFCs with respect to the X-WFCs distance allows to further quantify the degree of polarity of the G-X bonding, which results following the serie Ge-S (higher polarity) $>$ Ge-Se $>\mathrm{Ge}$-Te (lower polarity). These conclusions are found in sound agreement with the expected trends on the basis of Pauling electronegativity scale and polarizability values (see Table II).[49, 48] In particular, the $\omega$ values and the extension of its distribution with respect to X-WFCs distances allow to infer about the more softer (more polarizable, i.e. higher polarizability) nature of Te atoms with respect to $\mathrm{S}$ and Se atoms.

Once the surface model of the amorphous chalcogenide system of interest is produced by means of FPMD simulations, its optimized structure can be used to build a slit-like pore by introducing a proper vacuum space with respect to the pore width of interest. However, before switching to GCMC simulations for the study of gas adsorption and isotherms, a suitable set of atomic charges have to be assigned to the atoms of the host porous network. This step, together with the refinement of the chalcogenide glass' structure, represent the main challenge of the proposed computational procedure. Indeed, classical potentials are often unable to describe deviations from chemically ordered topologies occurring, for a given stoichiometry, in specific network structures (as glassy chalcogenides) made of interconnected tetrahedra. In the present work, we tentatively propose a set of atomic partial charges dependent on the local coordination. This approach is based on the exploitation of the charge equilibration method developed by Rappé and Goddard. This method is derived from atomic ionization energies and electron affinity values in order to compute partial atomic charges of the atoms with respect to covalent radii. Thanks to the fact that the charge on each atom is distributed over a Slater orbital having the size of the atom, the advantage of the Qeq method is to predict charges as a function of the local coordination environment.[47] This charge assignment results particularly suitable for the present case since it is shown to provide a physical picture of the glassy chalcogenide surfaces by describing the charge distribution as a function of the chemical order. The chemical order for gassy chalcogenides is not only sensitive to the 
composition but it can substantially differ from the perfect chemical order found in the crystalline parent phases. For instance, in the case of glassy $g-\mathrm{GeSe}_{2}$, we recall the perfect chemical order corresponds to the absence of any underor over-coordinated Ge or Se atoms. To be noted that the trend of partial charges obtained with the Qeq method are found to be in agreement with those obtained with the EQeq method[50] as well as with the Bader method[51, 52] on the basis of charge densities computed by FPMD-DFT data. Whereas the Lowdin,[53] Mulliken,[54] and the electrostatic potential-based (ESP)[55] methods lead to a nonzero net charge or to charges nearly insensitive to the coordination number.[30] The Qeq (EQeq) and Bader methods capture the effect of local coordination on the partial charges in chalcogenide materials. These methods provide reasonable partial charges for both the Ge and $\mathrm{X}$ atoms as their absolute charge increases with the coordination. However, the absolute charge values obtained by the Bader method are too large to be employed in classical simulations, conferring to the $\mathrm{Ge}$ and $\mathrm{X}$ atoms a nearly pure ionic character.Ori1 The charge-coordination correlation found in the Qeq(EQeq) method seems more appropriate to be employed for classical simulations such as GCMC or classical MD, presuming the potential parameters are consistent. Table I shows the atomic partial charges obtained by means of Qeq method for the $g$ - $\mathrm{GeS}_{4}, g-\mathrm{GeSe}_{4}$ and $g$-GeTe 4 systems as well as $g$ - $\mathrm{GeSe}_{2}$ and $g$-GeSe $\mathrm{G}_{9}$. With this methods over-coordinated $\mathrm{Ge}(\mathrm{X})$ atoms possess a large positive (negative) charge with respect to that corresponding to stoichiometric coordination. This is directly related to their higher valence state which, in a formalism purely based on formal ionic charges [cations $(\mathrm{Ge})$ and anions $(\mathrm{X})$ ], result in an increased charge localization. Likewise, the absolute charge value decreases descending along the element of the VI group of the periodic table for the counter-ion $\mathrm{X}\left(q_{S}>q_{S e}>q_{T e}\right)$. Along the Ge-Se serie, the absolute charge value for Ge sites increases with the Se:Ge ratio and it is counterbalanced by a decrease of the charge value of Se sties. Such method seems to be the best suited technique to describe changes in the valence (charge) state for different coordinations (e.g. structural order) as well as different chemical compositions and stoichiometry. However, it has to be underlined that it has not been demonstrated whether Qeq is as accurate as DFT-based methods reproducing QM energies nor that the predicted changes in polarization during dynamics agree with QM. Moreover, problem using this method could occur when high temperatures come into play or when extreme compositions are studied. With all these cautions in mind, the charges determined by Qeq method, in the presence of rationally physical situations, are reasonable and can be used to calculate the Coulomb interaction between atoms in (semi)empirical approaches.

\subsection{Grand canonical Monte Carlo simulations: quantitative prediction of gas adsorption isotherms}

Once a proper model of chalcogel pore is optimized by means of FPMD simulations, its structural model (i.e. atoms coordinates) and the corresponding charge atomic charge distribution (coordination dependent) can be employed to perform GCMC simulations in order to model the gas adsorption isotherm. The GCMC technique is a stochastic method applicable to a system having a constant volume $V$ (the pore with the adsorbed phase) in equilib- 
rium with an infinite reservoir of molecules imposing a chemical potential $\mu$ for each species $\left(\mathrm{N}_{2}\right)$ and temperature $T$. The absolute adsorption isotherm is given by the ensemble average of each number of adsorbate molecule as a function of the fugacity $f_{N_{2}}$ of the reservoir (the latter is determined from the chemical potential $\mu_{N_{2}}$. For the adsorbate, given the fugacities and temperatures considered in this work, the gas pressure $P$ is assumed to be equal to the fugacity $f_{N_{2}}$ (i.e. ideal gas assumption). We performed GCMC simulations of $\mathrm{N}_{2}$ adsorption at $T=77 \mathrm{~K}$ for chalcogel pores made of: $g-\mathrm{GeS}_{4}, g-\mathrm{GeSe}_{4}$ and $g-\mathrm{GeTe}_{4}$ as well as $g-\mathrm{GeSe}_{2}$ and $g-\mathrm{GeSe}_{9}$. We model the $\mathrm{N}_{2}$ adsorption isotherm between the 0.0 and the $\mathrm{N}_{2}$ saturation pressure $P_{0}$. Nitrogen adsorption at low temperature is a routine laboratory characterization technique of porous materials. For instance, the specific surface area of porous materials is usually assessed from adsorption experiments. Nitrogen was described using the model of Potoff and Siepmann (Trappe forcefield).[56] In this model, each $\mathrm{N}$ atom of the rigid $\mathrm{N}_{2}$ molecule is a center of repulsion and dispersion interactions via Lennar-Jones potential. In addition, each $\mathrm{N}$ atom bears a partial charge with $q_{N}=-0.482 e$, charges interacting through Coulombic forces. At the center of the N-N bond a partial charge $q=+0.964 e$ compensates the negative charge on the $\mathrm{N}$ atoms. Such charge distribution mimics the measured quadrupole moment of the $\mathrm{N}_{2}$ molecule. All the interactions between the atoms of the $\mathrm{N}_{2}$ molecules and the Ge and $\mathrm{X}$ atoms of the chalcogels were calculated by considering the intermolecular energy $U_{i j}(r)$ between two sites $(i$ and $j)$ as the sum of a Coulombic contribution and a pairwise-additive Lennard-Jones (LJ) 12-6 potential. In our simulations, the LJ cross interaction parameters $\left(\sigma_{i j}, \epsilon_{i j}\right)$ between unlike sites are calculated using the Lorentz-Berthelot mixing rules. The LJ parameters $\left(\sigma_{i j}, \epsilon_{i j}\right)$ for the Ge and X of the chalcogels were taken from Ref. [57]. The dispersive interactions were neglected beyond a cutoff of $10 \AA$. The electrostatic interactions were computed using the Ewald summation technique (the parameters were chosen so that the relative accuracy in the Coulomb energy calculation is $10^{-5}$ ). More details about the GCMC procedure and potential parameters employed in this study can be found in ref. [30].

\subsection{Detailed analysis of the gas/solid interface chemistry and computation of other properties.}

In order to refine the output configurations of the GCMC calculations of the systems made of $\mathrm{N}_{2}$ adsorbed in the chalcogels' pores, FPMD simulations can be employed with the purpose to further optimize the systems structure and chemistry as well as compute other properties of interest. As an example, once the new chalcogels systems are obtained containing different contents of gas molecules, MLWFs can be further computed to investigate into the details the interactions involved between the gas molecules and the solid glassy surfaces. Furthermore, MLWFs can be also employed to compute molecular dipoles to measure the induced polarization due to the interaction with the glassy solid surface. This exploitation of MLWFs allows to obtain a deeper insight into the interactions between the adsorbed gas molecules and the chalcogenide surfaces. Even for the case of apolar gas molecules for which the dipole moment averaged over time is expected to be zero, the instantaneous molecular distortions can result in non-zero instantaneous 
dipole moments $(\mu)$ and, in this respect, this analysis gives access to the local molecular polarization induced by the solid surface. Recently, we investigated the case of $\mathrm{CO}_{2}$ molecules adsorbed in a $g$-GeS $\mathrm{G}_{2}$ chalcogel slit-like pore.[58] In this case, for the system made of a monolayer of $\mathrm{CO}_{2}$ molecules physisorbed on a $g$-GeS $\mathrm{S}_{2}$ surface, the magnitude of the induced $\mu_{\mathrm{CO}_{2}}$ is found to be strongly dependent on the distance from the $g-\mathrm{GeS}_{2}$ surface. Close to the solid surface, the $\mathrm{CO}_{2}$ molecules show a large $\mu_{\mathrm{CO}_{2}}$ of about $\sim 0.51 \mathrm{D}$, while departing from the surface $\mu_{\mathrm{CO}_{2}}$ reaches values close to those expected for the $\mathrm{CO}_{2}$ in the gas phase $(\sim 0.1 \mathrm{D})$. The large variation of the dipole moments for the $\mathrm{CO}_{2}$ molecules in contact with the solid surface can be explained by the high polarizability of both Ge and S atoms of the $g-\mathrm{GeS}_{2}$ surface. $\mathrm{CO}_{2}$ molecules departing from the surface towards the empty space in the center of the pore restore almost completely the $\mu_{\mathrm{CO}_{2}}$ value typical of $\mathrm{CO}_{2}$ molecules in the gas phase. Similarly, Karseemeijer et al. [60] and Sun et al. [61] found values of $\mu_{\mathrm{CO}_{2}}$ of about $\sim 0.5 \mathrm{D}$ for $\mathrm{CO}_{2}$ molecules adsorbed on top of solid water. Interestingly, our calculated dipole moments values are similar to those of induced $\mu_{\mathrm{CO}_{2}}$ found for $\mathrm{CO}_{2}$ molecules adsorbed in a hydrated Ca-exchanged Montorillonite (in the range 0.4-1.0 D).[62] While the oxygen dipole moment coming from the electronic polarization in glassy silica was found weakly dependent from the local melt composition.[63]

\subsection{Models and methods details relevant to this work}

The bulk structural models of the chalcogenide glasses studied in this work were obtained by means of FPMD simulations following the typical melt-quenching technique. The details about the chalcogenide systems considered here are reported in Table I. The electronic structure was described within DFT by using as generalized gradient approximation (GGA) the Becke, Lee, Yang, and Parr (BLYP) exchange-correlation functional [37, 38] The BLYP functional was combined with the empirical Grimme dispersion correction for van der Waals (vdW) interactions.[59] This theoretical scheme has been successfully validated on a wide range of glassy chalcogenides. More specifics about the methodology used to obtain the different chalcogenide glasses can be found in the references reported in Table I. The surfaces models were initially built from the parent bulk models by removing the periodic boundary conditions along the $z$ direction. By inserting the slab having a thickness $\Delta_{z}$ in a simulation box of a size $h_{z}$, one defines a slit pore of width $H=h_{z}-\Delta_{z}$ via the use of periodic boundary conditions. More precisely, we took the pore width $(H)$ as the distance between the mean positions of the chalcogen and Ge atoms on the two opposite surfaces. We prepared systems with $H$ in between 2 to 4 in order to obtain $\mathrm{Ge}_{x} \mathrm{Ch}_{y}$ (with $\mathrm{Ch}: \mathrm{S}, \mathrm{Se}, \mathrm{Te}$ ) slit-like nanopores with different widths. A selection of the output configurations obtained from the GCMC simulations were further refined by means of FPMD simulations with applying a friction force on the atom dynamics (ion velocities scaled by a factor 0.95 at each step) in order to optimize the systems at $\mathrm{T}=0 \mathrm{~K}$. The surface models were then equilibrated by FPMD at finite temperature and volume. The above calculations on clusters were performed with the CPMD code. 

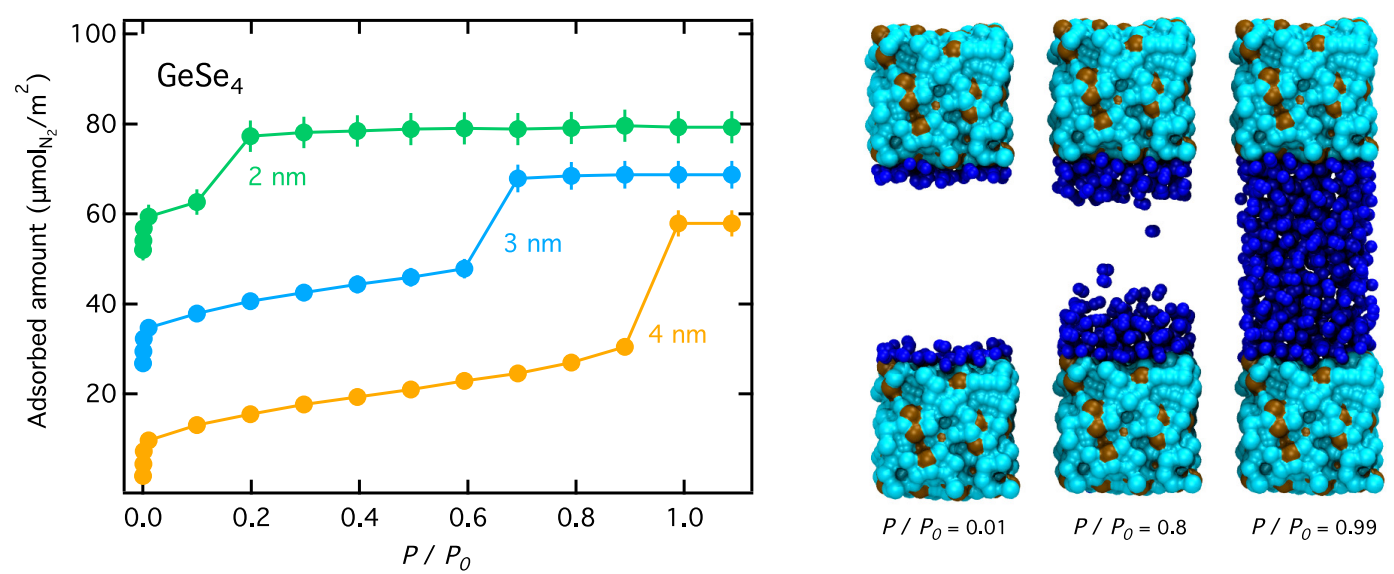

$P / P_{O}=0.01$

$P / P_{O}=0.8$

$P / P_{O}=0.99$

Figure 2: Left: $\mathrm{N}_{2}$ adsorption isotherms at $77 \mathrm{~K}$ for porous $g$-GeSe 4 with slit-like pores of width $H$ (indicated next to each adsorption isotherm). Adsorbed amounts are in $\mu \mathrm{mol} / \mathrm{m}^{2}$. For the sake of clarity, adsorption isotherms have been shifted up. Pressures are in relative units with respect to the bulk saturating pressure $P_{0}$ for $\mathrm{N}_{2}$ at $77 \mathrm{~K}$. Right: Typical molecular configurations for $\mathrm{N}_{2}$ adsorbed in $g$-GeSe $\mathrm{Gere}_{4}$ with $H=3.6$ 4: (from left to right) $P / P_{0}=0.01, P / P_{0}=0.80$, and $P / P_{0}=0.99$. The ochre and cyan spheres are the Ge and $\mathrm{Se}$ atoms of the chalcogenide surfaces, respectively. The blue spheres are the atoms of the $\mathrm{N}_{2}$ molecules.

\section{Case studies}

\subsection{Pore size effect}

Figure 2 shows the $\mathrm{N}_{2}$ adsorption isotherm for $g-\mathrm{GeSe}_{4}$ nanopores with $H=2,3$ and $4 \mathrm{~nm}$ computed at $77 \mathrm{~K}$. The data obtained for the three pores confirm the typical behavior observed in the experiments of adsorption/condensation in solid nanopores. At low pressures, the adsorbed amount increases in a continuous fashion upon increasing the pressure with the $\mathrm{N}_{2}$ forming an adsorbed film at the $g-\mathrm{GeSe}_{4}$ pore surface. Beyond adsorption of the first layer, the slope of the adsorption isotherm decreases once the pore gets filled along the multilayer adsorption regime. Then, at a pressure lower than the bulk saturating vapor pressure $P_{0}$, capillary condensation occurs. As expected on the basis of capillary condensation theories such as Derjaguin and Derjaguin-Broekhoff-De Boer model,[3, 5] the capillary condensation pressure increases with increasing the pore width $H$ : the condensation pressures for the nanopores with $H=2,3$, and $4 \mathrm{~nm}$ are $\sim 0.10 P / P_{0}, \sim 0.59 P / P_{0}$, and $\sim 0.89 P / P_{0}$, respectively. Figure 2 also shows typical molecular configurations of $\mathrm{N}_{2}$ molecules adsorbed at different relative pressures upon adsorption in the nanopore with $H=4$ $\mathrm{nm}$. Regardless of the pore width, the surface of $g-\mathrm{GeSe}_{4}$ nanopores is covered with a homogeneous film at the onset of capillary condensation. A discontinuous transition (i.e., capillary condensation) between the partially filled and completely filled configurations occurs when the adsorbed film becomes unstable, in line the results found for $g$-GeS and typical experimental data.[31] Note that for a proper quantitative comparison with experimental data this has to be normalized to the corrected Brunauer-Emmett-Teller (BET) [32] surface area. Generally, it is appropriate to correct the BET surface to account for the overestimate of the true geometrical surface areas by about $25-30 \%$.[4, 33] 


\subsection{Chemical stoichiometry - Se:Ge ratio effect}

Figure 3 shows the $\mathrm{N}_{2}$ adsorption isotherm for nanopores with $H=2 \mathrm{~nm}$ computed at $77 \mathrm{~K}_{\text {for }} \mathrm{Ge}_{x} \mathrm{Se}_{y}$ chalcogels with different Se:Ge ratio $\phi_{S e / G e}: 2,4$ and 9 for $g-\mathrm{GeSe}_{2}, g-\mathrm{GeSe}_{4}$, and $g-\mathrm{GeSe}_{9}$ respectively. The $\mathrm{N}_{2}$ adsorbed amount is normalized with respect to the pore surface area in order to take into account the different size of the simulated system (see Table I for details). We recall that the pore surface areas considered in this work allows to rule out any possible size effect, in accordance with the previous results obtained for the $g$-GeS $\mathrm{G}_{2}$ system. [31, 58] The data obtained for the three systems show a similar trend that follows the typical behavior observed for adsorption/condensation experiments in solid nanopores as discussed in the previous section. The onset of capillary condensation within the pore occurs at similar relative pressure for systems with $\phi_{S e / G e}=2$ and $4\left(P / P_{0} \sim 0.1\right)$ whereas for the $\phi_{S e / G e}=$ 9 system it occurs at larger relative pressure $\left(P / P_{0} \sim 0.2\right)$. Figure 3 (inset) shows the comparison between the $\mathrm{N}_{2}$ adsorption isotherms in the range $0.0<P / P_{0}<0.25$ for the three systems. The non-negligible difference found corresponds to a lower adsorbed amount of $\mathrm{N}_{2}$ at low pressure $\left(<0.15 P / P_{0}\right)$ for the $g$-GeSe ${ }_{9}$ system with respect to $g-\mathrm{GeSe}_{2}\left(\sim-11 \%\right.$ and $\sim-9 \%$ at $P / P_{0} \sim 0.01$ and $P / P_{0} \sim 0.1$ respectively $)$. This result suggests a weaker interaction between $\mathrm{N}_{2}$ molecules and the Se atoms of the chalcogel surface with respect to the interaction between $\mathrm{N}_{2}$ molecules and the Ge atoms. The stronger interaction between $\mathrm{N}_{2}$ and chalcogels with lower $\phi_{S e / G e}$ arises from the interplay between the electronegativity and electropositive charge of Ge atoms and its larger polarizability with respect to Se $\left(\alpha_{G e} \sim 42\right.$ vs $\left.\alpha_{S e} \sim 27\right)$.[48]. The specific gas interactions with these surfaces is a key factor determining the behavior of gas mixtures through porous structures. Our data is found in fair agreement with the experimental behavior for adsorption/condensation obtained by Armatas et al.[18] for $\mathrm{Ge}_{x} \mathrm{Se}_{y}$ chalcogels, where a system with a greater Se:Ge ratio is found to promote lower $\mathrm{N}_{2}$ adsorption at the same relative pressure $\left(\phi_{S e / G e} \sim 0.44\right.$ vs $\left.\phi_{S e / G e} \sim 0.21\right)$.

\subsection{Chemical composition - chalcogen effect}

Figure 4 shows the $\mathrm{N}_{2}$ adsorption isotherm for nanopores with $H=3 \mathrm{~nm}$ computed at $77 \mathrm{~K}$ for chalcogels made of different chalcogen counter-ions: $g$-GeS $4, g$-GeSe 4 , and $g$-GeTe 4 . The data obtained for the three systems show a similar trend that follows the typical behavior observed for adsorption/condensation experiments in solid nanopores as discussed in the previous section. The onset of capillary condensation within the pore occurs at similar relative pressure for the sulfide and selenide systems $\left(P / P_{0} \sim 0.6\right)$ whereas for the telluride system it occurs at larger relative pressure $\left(P / P_{0} \sim 0.8\right)$. Figure 3 (right panel) shows the comparison between the $\mathrm{N}_{2}$ adsorption isotherms in the range $0.0<P / P_{0}<0.25$ for the three systems. At a given relative pressure, the amount of $\mathrm{N}_{2}$ adsorbed on the chalcogel surface is found to be strongly related to the nature of the chalcogen counter-ion and following the series $g$-GeS $\mathrm{S}_{4}>$ $g-\mathrm{GeSe}_{4}>g-\mathrm{GeTe}_{4}$. For example, at relative pressure of $P / P_{0} \sim 0.3, g-\mathrm{GeSe}_{4}$ and $g-\mathrm{GeTe}_{4}$ show a lower adsorbed of 


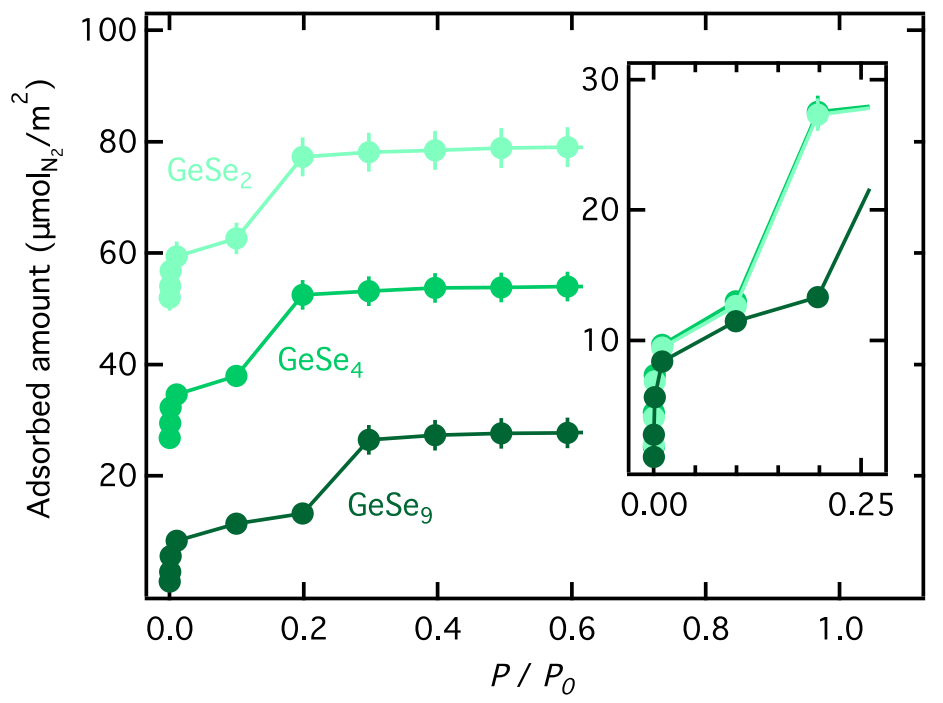

Figure 3: $\mathrm{N}_{2}$ adsorption isotherms at $77 \mathrm{~K}$ for slit-like pores with $H=2 \mathrm{~nm}$ made of $\mathrm{Ge}_{x} \mathrm{Se}_{y}$ chalcogels with different Se:Ge ratio $\phi S e / G e=2,4$ and 9 for $g$-GeSe $e_{2}, g$-GeSe 4 , and $g$-GeSe 9 respectively. For the sake of clarity, adsorption isotherms have been shifted up. Insets: zoom-in within the same $\mu \mathrm{mol}_{N 2} / \mathrm{m}^{2}$ scale at the low relative pressure $P / P_{0}$ range $\left(0.0<P / P_{0}<0.25\right)$.

$\mathrm{N}_{2}$ with respect to $g-\mathrm{GeS}_{4}(-5.9 \%$ and $-19.4 \%$, respectively). This behavior can be ascribed to the interplay between the electronegativity, charge and polarizability of the chalcogels constituents elements. It remains to be investigated if the interactions of different gases (such as $\mathrm{CO}_{2}, \mathrm{CH}_{4}$, and $\mathrm{H}_{2}$ ) with these porous glassy chalcogenides would follow the same trend.

\section{Conclusions and perspectives}

Realistic models of porous glassy chalcogenides were used to probe the viability of such a class of materials for adsorption applications. Using atomic-scale simulation based on first-principles molecular dynamics and grand canonical Monte Carlo simulations, we gained insights into the adsorption mechanisms for $\mathrm{N}_{2}$ molecules. Both the effects of pore size and chalcogenide chemistry (composition and stoichiometry) were investigated in order to gain fundamental understanding on the chalcogels gas adsorption properties and assess the versatility of computational modelling approach proposed. Our results are found to be in good agreement with available experimental data, confirming the ability of our methodology to produce realistic chalcogel models and predict their $\mathrm{N}_{2}$ gas adsorption performance. Owing to their high polarizability, the isosteric heats of adsorption show that chalcogenide surfaces have a stronger interaction with $\mathrm{CO}_{2}$ than with $\mathrm{CH}_{4}$ or $\mathrm{H}_{2}$. As a result, large selectivities were observed towards $\mathrm{CO}_{2}$ pointing to such materials as potential adsorbents for gas separation, such as land-gas separation or $\mathrm{CO}_{2}$ capture/sequestration. We also showed that selectivities can be accurately described using the ideal adsorbed solution theory (IAST) which predicts coadsorption isotherms based on pure component adsorption isotherms. Such a reliable theory allows avoiding to 

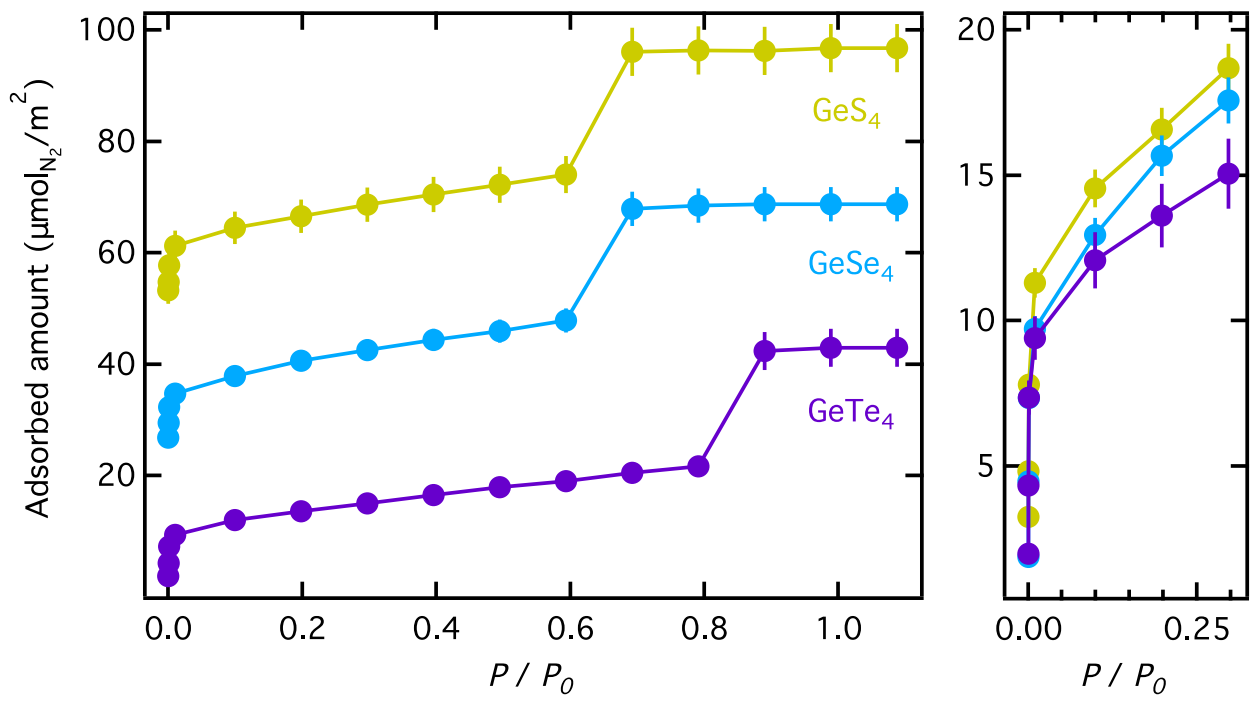

Figure 4: Left: $\mathrm{N}_{2}$ adsorption isotherms at $77 \mathrm{~K}$ for slit-like pores with $H=3 \mathrm{~nm}$ made of chalcogels based on different chalcogen element: $g$-GeS 4 , $g-\mathrm{GeSe}_{4}$, and $g-\mathrm{GeTe}_{4}$ respectively. For the sake of clarity, adsorption isotherms have been shifted up. Right: zoom-in within the same $\mu$ mol ${ }_{N 2} / \mathrm{m}^{2}$ scale at the low relative pressure $P / P_{0}$ range $\left(0.0<P / P_{0}<0.25\right)$.

measure coadsorption isotherms thereby considerably decreasing the cost and time needed to design efficient adsorbents based on porous chalcogenides. The present work shows that glassy porous chalcogenides are a valuable class of materials for gas adsorption. While further work is needed to clarify gas adsorption for different gas probes, these results shed light on the adsorption mechanisms for porous chalcogenides. Such an approach can be used for $a$ priori evaluation of their gas adosorption performances and pave the way for the design of chalcogenide-based adsorbents towards specific applications.

\section{Acknowledgement}

We acknowledge the Pôle HPC and Equipex Equip@ Meso at the University of Strasbourg and the Grand Equipement National de Calcul Intensif (GENCI) under allocation DARI-A0060807670. G.O. acknowledges the Seed Money program of Eucor - The European Campus (project MEDIA) for financial support.

\section{References}

[1] The Strategic Energy Technology Plan 2017, https://bit.ly/2kqMR6W.

[2] C. H. Lau, S. Liu, D. R. Paul, J. Xia, Y.-C. Jean, H. Chen, K. Shao and T.-S. Chung, Adv. Energy Mater, 2011, 1, 634-642.

[3] B. Coasne, A. Galarneau, R. J. M. Pellenq and F. Di Renzo Chem. Soc. Rev., 2013, 42, 4141-4171.

[4] B. Coasne and P. Ugliengo, Langmuir, 2012, 28, 11131-11141.

[5] B. Coasne NewJ.Chem., 2016, 40, 4078.

[6] X. Xu, C. Song, J.M. Andresen, B.G. Miller and A.W. Scaroni, Energy Fuels, 2002, 16, 1463-1469. 
[7] N.R. Stuckert and R.T. Yang, Environ. Sci. Technol., 2011, 45, 10257-10264.

[8] G. Li, P. Xiao, P. Webley, J. Zhang, R. Singh and M. Marshall, Adsorption, 2008, 14, 415-422.

[9] J. Merel, M. Clausse F. Meunier, Ind. Eng. Chem. Res., 2008, 47, 209-215.

[10] N. Du, H. B. Park, M. M. Dal-Cin and M. D. Guiver, Energy Environ. Sci., 2012, 5, 7306-7322.

[11] J. Lee, J. Kim and T. Hyeon, Adv. Mater., 2006, 18, 2073-2094.

[12] P. Billemont, B. Coasne and G. De Weireld, Langmuir, 2013, 29, 3328-3338.

[13] G. P. Hao, Z. Y. Jin, Q. Sun, X. Q. Zhang, J.-T. Zhang and A. H. Lu Energy Environ. Sci., 2013, 6, $3740-3747$.

[14] J.-R. Li, R. J, Kuppler and H.-C. Zhou, Chem. Soc. Rev., 2009, 38, 1477-1504.

[15] C. E. Wilmer, O. K. Farha, Y.-S. Bae, J. T. Hupp and R. Q. Snurr, Energy Environ. Sci., 2012, 5, 9849-9856.

[16] V. Stanić, A. C. Pierre and T. H. Etsell, J. Am. Ceram. Soc., 2000, 83, 1790-1796.

[17] K. K. Kalebaila, D. G. Georgiev and S. L. Brock, J. Non-Cryst. Solids, 2006, 352, 232-240.

[18] G. A. Armatas and M. G. Kanatzidis, Nature Mater., 2009, 8, 271-222.

[19] B. J. Riley, J. Chun, W. Um, W. C. Lepry, J. Matyas, M. J. Olszta, X. Li, K. Polychronopoulou and M. G. Kanatzidis, Environ. Sci. Technol., $2013,47,7540-7547$.

[20] Q. Lin, X. Bu, C. Mao, X. Zhao, K. Sasan and P. Feng, J. Am. Chem. Soc., 2015, 137, 6184-6187.

[21] H. Yang, M. Luo, X. Chen, X. Zhao, J. Lin, D. Hu, D. Li, X. Bu, P. Feng, and T. Wu, Inorg. Chem., 2017, 56, 14999-15005.

[22] R. G. Parr and R. G. Pearson, J. Am. Chem. Soc., 1983, 105, 7512-7516.

[23] M. G. Kanatzidis, Adv. Mater., 2007, 19, 1165-1181.

[24] M. Shafai-Fallah, A. Rothenberger, A. P. Katsoulidis, J. He, C. D. Malliakas and M. G. Kanatzidis, Adv. Mater., 2011, 23, 4857-4860

[25] E. Ahmed and A. Rothemberger, J. Mater. Chem. A, 2015, 3, 7786-7792.

[26] K. S. Subrahmanyam, C. D. Malliakas, D. Sarma, G. S. Armatas, J. Wu, and M. G. Kanatzidis, J. Am. Chem. Soc., 2015, 137, 13943-13948.

[27] B. J. Riley, D. A. Pierce, W. C. Lepry, J. O. Kroll, J. Chun, K. S. Subrahmanyam, M. G. Kanatzidis, F. K. Alblouwy, A. Bulbule, and E. M. Sabolsky, Ind. Eng. Chem. Res., 2015, 54, 11259-11267.

[28] S. Murugesan, P. Kearns and K. J. Stevenson, Langmuir, 2012, 28, 5513-5517.

[29] G. Leyral, M. Ribes, L. Courthéoux, D. Uzio and A. Pradel, Eur. J. Inorg. Chem., 2012, 31, 4967-4971.

[30] G. Ori, C. Massobrio, A. Bouzid, M. Boero and B. Coasne, Phys. Rev. B, 2014, 90, 045423.

[31] G. Ori, C. Massobrio, A. Pradel, M. Ribes and B. Coasne, Phys. Chem. Chem. Phys., 2016, 18, 13449-13458.

[32] S. Brunauer, P. H. Emmett and E. Teller, J. Am. Chem. Soc., 1938, 60, 309-319.

[33] A. Galarneau, H. Cambon, F. Di Renzo and F. Fajula, Langmuir, 2001, 17, 8328-8335.

[34] For this contribution as FPMD simulation method we adopted the Car-Parrinello approach [R. Car and M. Parrinello, Phys. Rev. Lett. 1985, 55, 2471] using the CPMD code [see http://www.cpmd.org/, copyright IBM Corp. 1990-2013, copyright MPI für Festkörperforschung Stuttgart 1997-2001.].

[35] G. Ori, A. Bouzid, E. Martin, C. Massobrio, S. Le Roux, M. Boero, Solid State Sci., 2019, 95, 105925.

[36] J.-Y. Raty, M. Schumacher, P. Golub, V.L. Deringer, C. Gatti, M. Wuttig, Adv. Mater., 2019, 31, 1806280.

[37] A. D. Becke, Phys. Rev. A, 1988, 38, 3098.

[38] C. Lee, W. Yang and R. G. Parr, Phys. Rev. B, 1988, 37, 785.

[39] J.P. Perdew, K. Burke, and M. Ernzerhof, Phys. Rev. Lett., 1996, 77, 3865.

[40] J.P. Perdew, K. Burke, and M. Ernzerhof, Phys. Rev. Lett., 1997, 78, 1396.

[41] A. Bouzid, C. Massobrio, M. Boero, G. Ori, K. Sykina, E. Furet, Phys. Rev. B, 2015, 92, 134208. 
[42] A. Bouzid, S. Le Roux, G. Ori, M. Boero, C. Massobrio, J. Chem. Phys., 2015, 143, 034504.

[43] A. Bouzid, S. Le Roux, G. Ori, C. Tugene, M. Boero, C. Massobrio, Molecular Dynamics Simulations of Disordered Materials: Springer Series in Materials Science., Ch. 12 2015,, 313-344.

[44] S. Le Roux, A. Bouzid, K.Y. Kim, S. Han, A. Zeidler, P.S. Salmon, C. Massobrio, Chem. Phys., 2016, 145, 084502.

[45] E. Lampin, A. Bouzid, G. Ori, M. Boero, C. Massobrio J. Chem. Phys., 2017, 147, 044504.

[46] C. Massobrio, E. Martin, Z. Chaker, M. Boero, A. Bouzid, G. Ori, Front. Mater., 2018, 5, 1-5.

[47] A. K. Rappé and W. A. Goddard III, J. Phys. Chem., 1991, 95, 3358-3363.

[48] P. Schwerdtfeger, J.K. Nagle, Mol. Phys., 2019, 117, 9-12.

[49] L. Pauling, The Nature of the Chemical Bond, th. ed. Cornell Univ. Press Ithaca, 1960.

[50] C. E. Wilmer, K. C. Kim, and R. Q. Snurr, J. Phys. Chem. Lett., 2012, 3, 2506.

[51] R.F.W. Bader, Atoms inMolecules: a Quantum Theory, Oxford Univ. Press, New York, 1990.

[52] C. Gatti and P. Macchi, Modern Charge-Density Analysis, Springer, Dordrecht, Heidelberg, London, New York, 2012.

[53] P.-O. L'owdin, J. Chem. Phys., 1950, 18, 365.

[54] R. S. Mulliken, J. Chem. Phys., 1955, 23, 1833.

[55] S. R. Cox and D. E.Williams, 2, 304 (1981). J. Comput. Chem., 1981, 2, 304.

[56] J. J. Potoff and J. I. Siepmann, AIChE J., 2001, 47, 1676-1682.

[57] S. L. Mayo, B. D. Olafson and W. A. Goddard, J. Phys. Chem., 1990, 94, 8897.

[58] Z. Chaker, A. Bouzid, B. Coasne, C. Massobrio, M. Boero, G. Ori, J. Non-Cryst. Solids, 2018, 498, $288-293$.

[59] S. Grimme, J. Compt. Chem., 2006, 27, 1787-1799.

[60] L.J. Karssemejier, G.A. Wijs, H.M. Cuppen, Phys. Chem. Chem. Phys., 2014, 16, 15630.

[61] Z. Sun, D. Pan, L. Xu, E. Wang, Proc. Natl. Acad. Sci. U.S.A., 2012, 109, 13177-13181.

[62] M.-S. Lee, B. P. McGill, R. Rousseau, V.-A.Glezakou, J. Phys.Chem. C, 2017, 122, 1125.

[63] R. Vuilleumier, N. Sator, B. Guillot, J. Non-Cryst. Solids, 2011, 357, 2555. 